\title{
The Global Conference on Agricultural Research for Development
}

A major event during these last weeks was the Global Conference on Agricultural Research for Development, GCARD, which was held in Montpellier, France, from 28th to 31st of March, 2010. This conference brought together more than 1,000 researchers, policymakers, farmers, donors, and members of civil society from every region of the world. The aim was to develop a new agricultural research for development (AR4D) architecture that is geared toward reducing both hunger and poverty through innovative approaches far from business as usual. It is the first time all key players, from farmers to donors, have gathered to iron out a roadmap for AR4D.

Failure to prioritize agriculture and rural development at the same level as other sectors such as health and education has left many developing countries with gaps in the capacity needed to meet the Millennium Development Goal of reducing hunger and poverty. It has also left them unprepared for coping with rapid climate change and the population explosion expected to occur by 2050. Some key issues for change were highlighted. One of them is a greater involvement of the civil society and private sector in the AR4D process. Food providers and consumers must be at the center and in the governance of agricultural research at the international, regional and national levels.

Another one is the role of women. A key sentence was: "Investing in gender is non-negotiable". If not, a key leverage will not be activated for effective development, as women are the key actors for a lot of its components including education, nutrition and health, amongst others.
To address such a challenge, all the research community must be mobilized globally, and a keyword is "partnership". International centers of the CGIAR must work much more closely with national institutions; industrialized countries must interact much more with developing countries on a mutual interest basis; farmers, civil society, the private sector and consumers must be an integral part of the process.

GCARD is the first step of a long trip. In order to move from declarations to actions, it is necessary to keep the "spirit of Montpellier" and continue to build on it.

But it is clear that fruits and vegetables, although claimed as essential for nutrition and health, and key drivers for income generation, employment and livelihood, are still too often relegated behind staple food in the final action plans, and this conference will not have made it possible to reverse this tendency. It is time to highlight their key role for development if we do not want to be condemned to saying in a few decades that failure to prioritize fruits and vegetables at the same level as staple food has left many developing countries with gaps in the capacity needed to correctly address nutrition deficiencies and chronic diseases, lacking at the same time income generation opportunities.

Dr. Jacky Ganry Chairman of the Section on Tropical and Subtropical Fruits ISHS 


\section{La Conférence Mondiale sur la Recherche Agronomique pour le Développement}

Un événement important de ces dernières semaines a été la Conférence Mondiale sur la Recherche Agronomique pour le Développement, GCARD, qui s'est tenue à Montpellier, France, du 28 au 31 mars 2010. Cette conférence a rassemblé plus du 1000 chercheurs, décideurs, agriculteurs, donateurs, et membres de société civile de toutes les régions du monde. L'objectif en était l'élaboration d'une nouvelle architecture de la recherche agronomique pour le développement apte à mieux contribuer à la réduction de la faim et de la pauvreté par des approches novatrices loin de certaines pratiques habituelles. C'est la première fois que tous les acteurs clés, des agriculteurs aux donateurs, se sont réunis pour élaborer une feuille de route visant la recherche agronomique pour le développement.

Faute d'avoir donné une priorité à l'agriculture et au développement rural à la hauteur des autres secteurs, comme la santé et l'éducation, nombre de pays en voie de développement n'ont pas pu surmonter leurs lacunes dans leur capacité à atteindre les objectifs du Millénaire pour réduire la faim et la pauvreté ou pour faire face au changement climatique rapide et, ainsi, affronter l'explosion démographique prévue d'ici 2050. Quelques facteurs de changement essentiels ont été mis en avant. L'un d'entre eux concerne la participation de la société civile et du secteur privé dans le processus de recherche agronomique pour le développement. Les pourvoyeurs de nourriture et les consommateurs doivent être au centre et participer à la gouvernance de la recherche agronomique aux niveaux international, régional et national.

Un autre élément essentiel concerne le rôle des femmes. Une phrase marquante a été : "Investir dans le genre n'est pas négociable." Sans quoi un levier majeur ne sera pas activé pour un développement efficace, car les femmes sont les actrices principales pour beaucoup de ses composantes dont l'éducation, la nutrition et la santé notamment.

Pour relever un tel défi toute la communauté de la recherche doit être mobilisée au niveau mondial et un mot-clé est " partenariat ". Les centres internationaux du CGIAR doivent travailler beaucoup plus étroitement avec les structures nationales; les pays industrialisés doivent beaucoup plus interagir avec les pays en développement sur une base d'intérêt mutuel ; les agriculteurs, la société civile, le secteur privé et les consommateurs doivent faire partie intégrante du processus.

La GCARD n'est qu'une première étape d'un long parcours. Pour passer des déclarations aux actions il est nécessaire de préserver " l'esprit de Montpellier " et de continuer à construire à partir de lui.

Mais il est clair que les fruits et les légumes, même s'ils sont considérés comme essentiels pour la nutrition et la santé et comme sources de revenus, d'emploi et de qualité de vie, sont encore trop souvent relégués derrière les aliments de base dans les plans d'action finaux, et cette conférence n'aura pas permis d'inverser la tendance. Il est temps de mettre en lumière leur rôle essentiel pour le développement si nous ne voulons pas être condamnés à dire dans quelques décennies que faute d'avoir donné la priorité aux fruits et aux légumes à la hauteur des aliments de base, nombre de pays en voie de développement n'ont pas pu surmonter leurs lacunes pour correctement surmonter les déficiences nutritionnelles et des maladies chroniques, manquant en même temps des opportunités d'accroître leurs revenus.

Dr. Jacky Ganry

Président de la section Fruits tropicaux et subtropicaux

ISHS 